\title{
Single layer a-Si GRIN waveguide coupler with lithographically defined facets
}

\author{
S. Janz, B. Lamontagne, A. Delâge, A. Bogdanov, D.-X. Xu, K.P. Yap \\ Institute for Microstructural Sciences, National Research Council Canada, \\ 1200 Montreal Road, Ottawa, Ontario, Canada, K1A 0R6
}

\begin{abstract}
The fabrication of a monolithically integrated single layer graded index (GRIN) layer waveguide coupler, including a process for creating lithographically defined optical facets, is described. The coupling efficiency of GRIN couplers of varying lengths is measured, yielding an optimal coupling length of $\mathbf{1 5}$ $\mu \mathrm{m}$ for a single $3 \mu \mathrm{m}$ thick a-Si layer on a $0.8 \mu \mathrm{m}$ Si waveguide.
\end{abstract}

\section{INTRODUCTION}

Coupling light into small high index contrast waveguides remains one of the more challenging aspects of implementing microphotonic waveguide devices in high index material systems such as silicon-on-insulator (SOI). Many approaches involve fabricating two dimensional structures such as inverse tapers [1,2] or photonic crystals and gratings [3], using e-beam or deep-UV lithography. Gray scale lithography may also be used to create vertically tapered waveguide couplers [4]. The use of planar graded index lens structures for coupling has also been suggested, using a digital index profile [5] and continuous index profile free standing planar lens structures fabricated using $\mathrm{a}-\mathrm{Si}_{\mathrm{x}} \mathrm{O}_{\mathrm{y}}[6]$. Recently our group has presented theoretical results demonstrating that a graded-index (GRIN) half-lens structure grown directly on the SOI waveguide surface as in Fig. 1 may be used to couple light into a thin SOI waveguide [7]. The spatial resolution required to pattern these monolithic couplers is approximately $\pm 1 \mu \mathrm{m}$, well within the range of standard photolithographic tools. The necessary refractive index values to create the index lens profile $(3.3<\mathrm{n}<3.47$ at $\lambda=1550 \mathrm{~nm}$ ) can be obtained using well controlled PECVD deposition of amorphous silicon (a-Si).

This planar waveguide GRIN structure operates in much the same way as a conventional cylindrical GRIN lens. The input light periodically converges in the SOI waveguide core, and then diverges as shown in Fig. 1(b). A coupler is formed by removing the GRIN layers beyond the first focal point of the coupler as in Fig. 1(a). The refractive index of an ideal planar GRIN waveguide lens decreases with a quadratic dependence on distance from the waveguide surface, but much simpler GRIN index profiles consisting of a three or two uniform layers with a stepwise decreasing index profile can be almost as effective. Even the simplest case of a single uniform layer of slightly lower index than the waveguide core, as in Fig. 1(b), can increase the optical power coupled into a small SOI waveguide from a fiber or large input beam by several times.
In this paper we review our progress towards the experimental demonstration of monolithic GRIN couplers based on a-Si layers grown on SOI waveguides. Fabricating a GRIN coupler involves two key steps. The first is to deposit and pattern the a-Si GRIN layers on the SOI waveguide to form the coupler. The second is to create a lithographically defined input facet so that the correct coupler length is produced. Given relatively large uncertainty in final facet position after dicing and polishing, an alternative method to dicing and facet formation must be used. This facet positioning problem is of course not unique to the GRIN couplers, since it is also required for other coupling and hybrid integration technologies.

\section{EXPERIMENT}

The SOI waveguide with an a-Si coupler is shown in a schematic cross-section in Fig. 1. The SOI waveguides were formed in a $0.8 \mu \mathrm{m}$ silicon layer on a $0.4 \mu \mathrm{m}$ buried $\mathrm{SiO}_{2}$ layer. Ridges were etched using reactive ion etching, to an etch depth of approximately half the Si layer thickness. The ridge waveguide width was $2.0 \mu \mathrm{m}$, with $400 \mu \mathrm{m}$ long adiabatic tapers expanding to a $10 \mu \mathrm{m}$ wide waveguide at the input and output facets. A $0.5 \mu \mathrm{m}$ thick layer of $\mathrm{SiO}_{2}$ was deposited on top of the SOI waveguide layer. The waveguide coupler sections were defined by etching a window in this $\mathrm{SiO}_{2}$ layer over the waveguide adjacent to the eventual input facet position. Finally a $3 \mu \mathrm{m}$ layer of a-Si was deposited by plasma enhanced chemical vapour deposition (PECVD) over the entire wafer, and subsequently removed everywhere except over the coupler sections. The remaining a-Si regions overlap the boundaries of the oxide window so that the coupler length is defined by the oxide window dimensions, as shown in Fig. 1(a). Couplers of different lengths from 5 $\mu \mathrm{m}$ to $200 \mu \mathrm{m}$ were fabricated, so that the dependence of coupling efficiency on coupler length could be measured. The refractive index of the PECVD a-Si was measured using ellipsometry at a wavelength of $\lambda=1550 \mathrm{~nm}$. The refractive index over the die area showed some spatial variation, ranging from 3.36 to 3.40 . This is index range is less than the $\mathrm{Si}$ index of 3.47 , as required for effective GRIN lens focusing.

Although the monolithic GRIN coupling scheme does not require specialized lithography, it is important that the final coupler length be within a few microns of the optimum value. To achieve this objective, a lithographically defined 


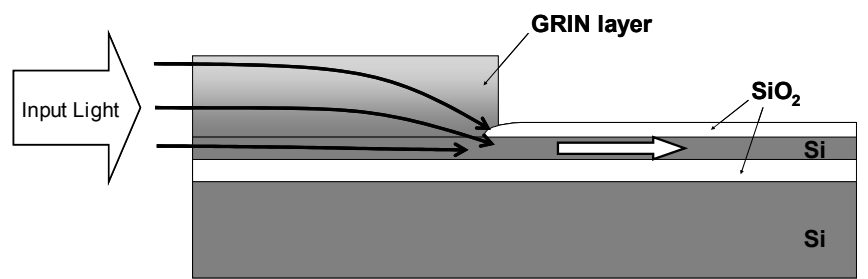

(a)

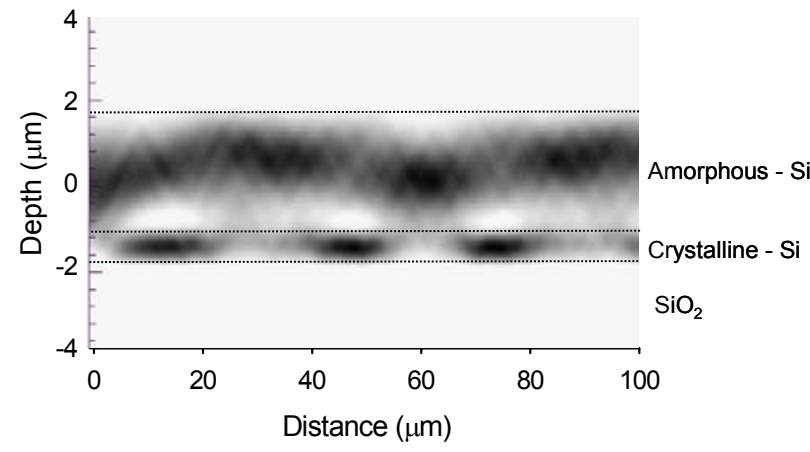

(b)

Fig. 1(a) Schematic of the monolithic GRIN coupler structure, and (b) the calculated intensity distribution in a waveguide consisting of a $0.8 \mu$ m Si waveguide core $(\mathrm{n}=3.47)$ and a uniform $3 \mu \mathrm{m}$ thick a-Si coupler layer $(\mathrm{n}=3.365)$.

inductively coupled plasma (ICP) etch process was developed to fabricate the input and output facets. The facet fabrication process involves two trench etching steps. First a shallow $5 \mu \mathrm{m}$ deep trench is formed to define the end facet of the waveguide. The relatively shallow etch and short etching time ensures that the optical facets are vertical, and also minimizes roughness arising from the etch attacking the vertical face of the facet. A thin layer of aluminum is then deposited by thermal evaporation as a protection layer for the facet face, before resuming a second etch to form a final 70 $\mu$-deep trench. A cryogenic $\mathrm{O}_{2}-\mathrm{SF}_{6}$ process is used to carry out the deep etch. The deep trench is used as a guide to the final dicing and cleaving of waveguide device, and ensures clearance for optical fiber to be brought into close proximity of the waveguide end facet. Scanning electron microscope images such as the one shown in Fig. 2 confirm that the integrity of the SOI waveguide end surfaces is preserved after the deep facet etching. Measurements of total insertion loss on similar waveguide devices with etched facets and polished facets show that both processes produce facets of comparable optical quality. The facet etching

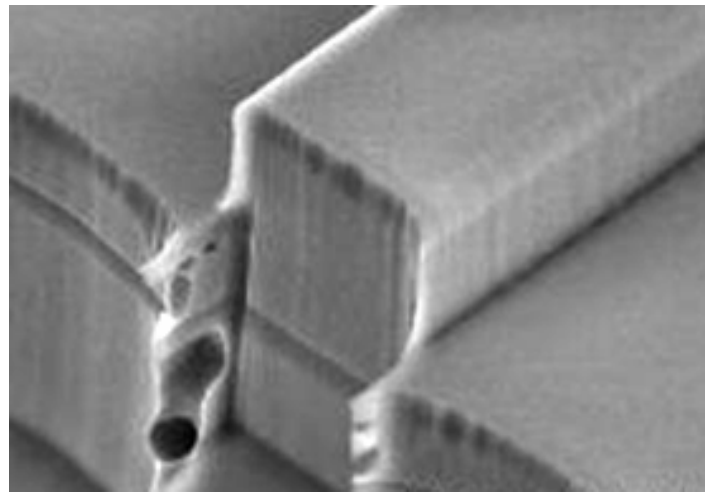

Fig. 2 A scanning electron microscope image of a lithographically defined ICP etched waveguide facet as described in text. The waveguide ridge is approximately $2 \mu \mathrm{m}$ wide technique can be used to fabricate coupling facets to within $\pm 1 \mu \mathrm{m}$ of a specified distance from the GRIN coupler termination point. Measurements of the relative coupling efficiency of GRIN couplers were carried out using a broadband erbium-doped fiber source $(\lambda \sim 1525-1560 \mathrm{~nm})$ coupled to the waveguide input facet from the cleaved end of a polarization maintaining fiber. Fig. 3 shows the measured output intensity for a series of waveguides with increasing coupler length from $0 \mu \mathrm{m}$ to $80 \mu \mathrm{m}$. The coupling efficiency increases by approximately four times at the optimal coupling length near $15 \mu \mathrm{m}$. The combined polarization dependent loss of the optimal coupler and contiguous waveguide is better than $-0.4 \mathrm{~dB}$.

The expected field evolution and coupling efficiency for the experimental structure were calculated using semivectorial beam-propagation method (BPM) simulations, shown in Fig. 1(b). The calculated variation of coupling

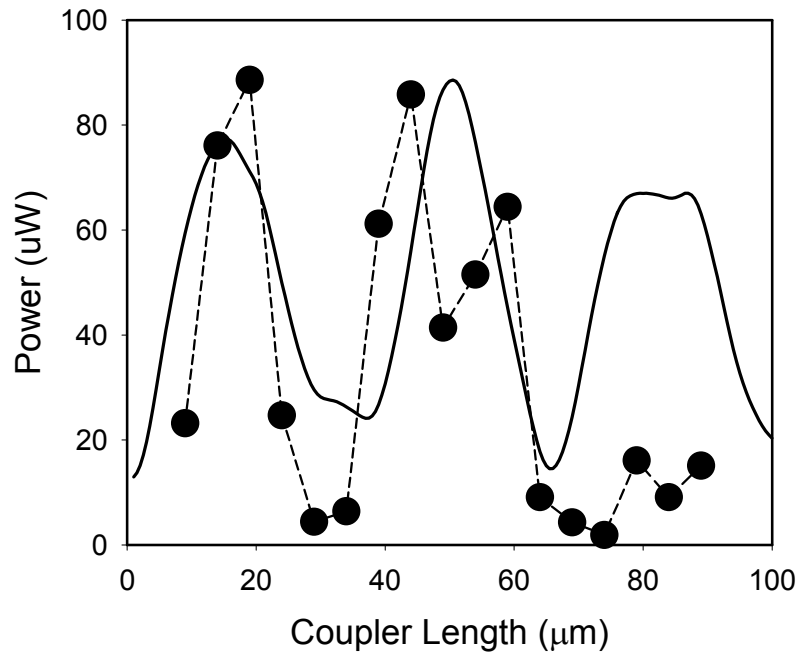

Fig. 3 The measured variation of the transmission through SOI waveguides with a-Si GRIN coupler length. 
efficiencies with coupler length is also shown in Fig. 3, for an a-Si refractive index of $n=3.365$. The experimental and theoretical coupling efficiency results are in good qualitative agreement, with respect to both the coupling periodicity and variation of coupling magnitude. A Gaussian mode centered on the waveguide formed by the combined $\mathrm{Si}$ layer and a-Si layer is used as the launched field for the simulations shown in Fig. 1(a) and Fig. 3. Although previous theoretical work [7] has shown that an optimized quadratic index profile GRIN coupler or three layer GRIN structure is not very sensitive to fiber misalignment and mode shape, BPM simulations for the single layer structure investigated here show that the single layer coupler can be more sensitive to details of input mode shape. Nevertheless, the periodicity and peak coupling efficiencies remain approximately the same, regardless of launched mode.

\section{SUMMARY}

We have described the fabrication and characterization of a monolithically integrated GRIN coupler formed using a single uniform a-Si layer deposited on a $0.8 \mu \mathrm{m}$ thick SOI waveguide. To ensure a specific coupler length can be fabricated, a lithographically defined process for creating waveguide facets and dicing the wafer at a precise location has been developed. The measured variation of coupling efficiency with GRIN coupler length is in good qualitative agreement with theoretical calculations.

\section{REFERENCES}

[1] V.R. Almeida, R.R. Panepucci, and M. Lipson, "Nanotaper for compact mode conversion", Opt. Lett. 28, pp. 1302-1304 (2003).

[2] K.K. Lee, D.R. Lim, D. Pan, C. Hoepfner, W.-Y. Oh, K. Wada, L.C Kimmerling, K.P. Yap and M.T. Doan, "Mode transformer for miniaturized optical circuits," Opt. Lett. 30, pp. 498-500 (2005).

[3] G.Z. Masanovic, V.M.N. Passaro, and G.T. Reed, "Dual gratingassisted directional coupling between fibers and thin semiconductor waveguides", IEEE Phot. Technol. Lett. 15, pp. 1395-1397 (2003).

[4] A. Sure, T. Dillon, J. Murakowski, C. Lin, D. Pustai, and D. Prather, "Fabrication and characterization of three-dimensional silicon tapers," Optics Express 11, pp. 3555-3561 (2003).

[5] C. Manolatou, and H.A. Haus, "Passive components for Dense Optical Integration", (Kluwer Academic Publishers, Boston, 2002).

[6] K. Shiraishi, C.S. Tsai, H. Yoda, and K. Minagawa, "A micro-GRIN slab tip fopr integrating coupling between superfine-core waveguides and single mode fibers", Proceedings of CLEO/Pacific RIM 2003, CDROM (IEEE, Piscataway, NJ, 2003).

[7] A. Delâge, S. Janz, D.-X. Xu, D. Dalacu, B. Lamontagne, and A. Bogdanov, "Graded-index coupler for microphotonic waveguides," SPIE Proc. Vol. 5577, pp. 204-212 (SPIE, Bellingham,WA, 2004). 